Open Science as a Better Gatekeeper for Science and Society: A Perspective from Neurolaw

\title{
Chuan-Peng $\mathrm{Hu}$
}

Deutsches Resilienz Zentrum (DRZ) \& Neuroimaging Center (NIC), University Medical Center

of the Johannes Gutenberg University, Mainz, Germany.

Xiaoming Jiang

Department of Psychology, Tongji University

Ricky Jeffrey

Language Center, International Campus, Zhejiang University

Xi-Nian Zuo

CAS Key Laboratory of Behavioral Science, Institute of Psychology

Author Note:

Chuan-Peng Hu, Neuroimaging Center (NIC), University Medical Center of the Johannes Gutenberg University, 55131 Mainz Germany, email: hcp4715@gmail.com;

Xiaoming Jiang, Department of Psychology, Tongji University, Shanghai, 200092, China, email: xiaoming.jiang@tongji.edu.cn

Ricky Jeffrey, Language Center, International Campus, Zhejiang University, Haining, 314400, China, email: rickyjeffrey@intl.zju.edu.cn

Xi-Nian Zuo, CAS Key Laboratory of Behavioral Science, Institute of Psychology, Beijing 100101, China, email: zuoxn@ psych.ac.cn

Please correspond to Chuan-Peng Hu (hcp4715@gmail.com;) or Xi-Nian Zuo

(zuoxn@psych.ac.cn) 
In 2011, a court near Milan, Italy, reduced the sentence of a convicted murderer from life to 20 years in jail, after considering results from neuroimaging and genetic tests. These tests had

3 been used to convince the judge that the trial's female defendant, Stefania Albertani, was

4 suffering from partial mental illness (Feresin, 2011). The structural MRI images showed that

5 Albertani's grey matter volume was different from the average volume of a randomly-selected

6 group - 10 healthy women with no history of mental or neurological disorders - in brain areas

7 including the anterior cingulate gyrus (ACC) and insula. The expert testimony concluded that

8 "these alterations (of brain structures) have to be considered in causal relation to the psychiatric

9 symptomatology of the murderer."

10 This is one of the many cases where structural or functional brain imaging results have

11 been cited as evidence in the courtroom, alongside other evidence, in order to support claims that

12 a defendant was mentally ill (Farahany, 2016). Which findings from cognitive neuroscience can

13 be used as evidence in the courtroom (in the form of expert testimony) is thus a central question

14 of the interdisciplinary area between cognitive neuroscience and law, known as 'neurolaw' or

15 'neuroscience and the law', e.g. (Gazzaniga, 2008; Hu, Deng, Zhou, \& Deng, 2011; Jones,

16 2004). Judges must assess the scientific validity and reliability of the cited scientific evidence in

17 order to decide its admissibility. This constitutes a significant challenge, since judges may have

18 limited training in science (Grove, Barden, Garb, \& Lilienfeld, 2002).

19 The recent reproducibility crisis in science, affecting medical science, neuroscience, and

20 behavioral science among other fields, has made this situation worse (Begley \& Ellis, 2012; Hu

21 et al., 2016; Open Science Collaboration, 2015; Szucs \& Ioannidis, 2017). (For a comprehensive

22 reference list, please see the reading list compiled by Brent Robert and Dan Simons:

23 https://osf.io/xunpj/). Studies suggest that the false positive rate in the psychological and 
1 neuroscience literature is surprisingly high (Szucs \& Ioannidis, 2017). The legal system, which

2 relies on the scientific community to provide scientific evidence (see Figure 1), must thus now

3 consider how to deal with this low reproducibility rate in published results (Chin, 2014).

4

11 from the US legal system offers one example. It requires judges to evaluate the expert evidence

12 (scientific evidence included) when deciding whether this evidence is admissible, and has been

13 intensively studied both in the United States (Faigman \& Monahan, 2009) and in other countries

14 (Page, Taylor, \& Blenkin, 2011).

15 The standard requires that judges evaluate the validity of scientific evidence based on

16 four criteria: (i) Is the opinion testable and has it been tested? (ii) Is the error rate associated with

17 the technique or opinion acceptable? (iii) Has the basis for the opinion survived the peer review

18 process and been published? (iv) Is the technique or procedure for generating the opinion

19 generally accepted among scientists in that field? 
The Daubert standard suggests that, even though the judges are required to assess the scientific evidence themselves, they still rely heavily on input from the scientific community.

3 Standards (iii) and (iv) require that the theory or technique behind the relevant scientific

4 evidence has been approved by scientists. Thus, the scientific community acts as a gatekeeper for 5 science in the first stage (Fig. 1).

According to the Daubert standard, if scientific results are flawed and yet nonetheless

7 published, judges may have difficulty in excluding any evidence based on such results. To

8 illustrate this impact of published but irreproducible results on the admissibility of scientific

9 evidence, we can consider the Albertani case mentioned earlier. In this case, as in many others

10 (Farahany, 2016; Hughes, 2010), the observation of abnormal brain structures in a single MRI

11 scan was cited as evidence in expert testimony to infer the mental state of the offender.

12 Before analyzing how irreproducible research compromises the Daubert standard, we

13 need to first assume that claims from experts are testable and have been tested, i.e. that the

14 relationship between the ability to control one's behavior and the brain structure underlying this

15 ability can be tested. At first glance, many published studies seem to report cognitive

16 neuroscientific findings that are linked to defendants' self-control. For example, behavioral

17 flexibility, the ability to control one's attention, is a cognitive process frequently studied in

18 psychology and cognitive neuroscience, and is supposedly related to self-control. Studies found

19 that variation in behavioral flexibility is correlated with the thickness of grey matter in the lateral

20 prefrontal cortex and ACC (Westlye, Grydeland, Walhovd, \& Fjell, 2011). However, the

21 concepts of self-control in law and in psychology and cognitive neuroscience are not always the

22 same thing: while self-control in the law is associated directly with behaviors, criminal behaviors

23 in particular, self-control in psychological and cognitive neuroscience studies is operationalized 
1 with some specific task that might not be directly associated with behaviors (Buckholtz \&

2 Faigman, 2014; Saunders, Milyavskaya, Etz, Randles, \& Inzlicht, 2017). In other words, when

3 considering expert opinion on offenders' ability to exert self-control, at worst this opinion may

4 not even be testable, and at best it has not yet been tested.

5 Further, even if we assume that the operationalized tasks in psychology and neuroscience

6 are indeed related to self-control ability in the legal sense, as many lawyers assume when citing

7 brain imaging results, irreproducible results can still compromise the Daubert standard.

First, it is difficult to estimate the error rate based only on published papers, hence

9 compromising the second standard of Daubert. The error rate indicated in the Daubert standard

10 is in most cases interpreted as statistical error and method error (Christensen, Crowder, Ousley,

$11 \&$ Houck, 2014). While the former error mainly refers to the differences between the predicted

12 and the real values, estimated by measure of uncertainty (e.g. standard deviation), the latter error

13 is more inherent to the methods itself (i.e. the sensitivity, meaning the proportion of true

14 positives that are correctly identified by the method, and specificity, meaning the proportion of

15 true negatives that are correctly identified by the method) (Trafimow \& Earp, 2017). The

16 sensitivity of a brain imaging technique in detecting the relationship between brain structures and

17 behavior might be low if the relationship reported in one brain imaging study cannot be

18 reproduced in a direct replication. Indeed, the above mentioned study about the relationship

19 between behavioral flexibility and the volume thickness of grey matter in the lateral prefrontal

20 cortex and ACC (Westlye et al., 2011) has not been replicated successfully (Boekel et al., 2015).

21 Further, it would be impossible to estimate the sensitivity and specificity of using the brain

22 imaging technique to detect brain-behavior relationship if the publicly reported results have been

23 selected by publication bias (Ioannidis, Munafò, Fusar-Poli, Nosek, \& David, 2014). The 
1 existence of publication bias means that the literature does not reflect positive and negative

2 results in an objective way. For example, on the relationship between brain structure and self-

3 control, if only positive results have been published, we would not be able to evaluate the

4 reliability and robustness of these results (Ioannidis et al., 2014).

5 Second, the peer review and publication standard of Daubert is compromised by

6 publication bias, which has inflated the false positive rate in the cognitive neuroscience literature

7 (Fusar-Poli et al., 2014; Ioannidis et al., 2014). Recent studies have revealed that the flexibility

8 researchers have when selecting their own analytical approach to generate results is of a high

9 level (Carp, 2012; Chen, Lu, \& Yan, 2018; Simmons, Nelson, \& Simonsohn, 2011).The

10 combination of researchers' flexibility and publication bias may have resulted in inflation of

11 false positives in the cognitive neuroscience literature. In this situation, the published results are

12 untrustworthy, and therefore compromise the peer review and publication standard.

13 Third, in the field of cognitive neuroscience, especially for neuroimaging studies, there is

14 no generally accepted standard for methods. Almost every paper reports having used a different

15 workflow to process the data (Carp, 2012; Yeung, 2018). The standardization of data processing

16 has not received sufficient recognition, though a recent publication calls a consensus to be

17 developed in data processing routine (Nichols et al., 2017). Given this proliferation of different

18 research procedures, it is difficult to say that any procedure referenced in a legal case is generally

19 accepted among the community of cognitive neuroscientists or not.

20 In sum, the reproducibility problems in cognitive neuroscience undermine the legal

21 gatekeeping process (the Daubert standards). The situation becomes even worse if both the

22 experts (clinical psychologists or neuroscientists) and the judges lack necessary training in

23 statistics (Lyu, Peng, \& Hu, 2018; Tavris, 2014). 


\section{The open science movement can benefit the gatekeeper system}

Fortunately, the scientific community has taken actions to address the credibility crisis

3 caused by the reproducibility issue. The most prominent approach is the open science movement,

4 which is characterized by openness, transparency, and reproducibility in scientific research

5 practice (Munafò et al., 2017). Direct replications are encouraged (Zwaan, Etz, Richard E., \&

6 Donnellan, 2018); pre-registration before collecting data is becoming a new norm, e.g.

7 (Chambers, Dienes, McIntosh, Rotshtein, \& Willmes, 2015; Nosek, Ebersole, DeHaven, \&

8 Mellor, 2018); open data, materials and code are required (Nosek et al., 2015); new inferential

9 statistical techniques are introduced and new tools developed, e.g. (Hu, Kong, Wagenmakers,

10 Ly, \& Peng, 2018; Wagenmakers, Wetzels, Borsboom, \& van der Maas, 2011); and standard

11 protocols to analyse data and report results are proposed, e.g. (Liu et al., 2018; Nichols et al.,

12 2017; Poldrack et al., 2017; Shamseer et al., 2015). In sum, the open science movement has

13 started to clean out the influential but irreproducible studies, and increase the transparency, rigor 14 and reproducibility of new studies (Milham et al., 2018; Nosek et al., 2015; Nosek, Ebersole,

15 DeHaven, \& Mellor, 2017), so as to enhance science's ability to describe, explain and predict.

16 The benefit of the open science movement to science itself has been discussed at great

17 length recently, and is beyond the scope of the current discussion (see: https://osf.io/xunpj/ for a

18 comprehensive reference list). Below we will focus on how the open science movement can

19 benefit the gatekeeping process for admitting scientific evidence into the courtroom.

First, the open science movement may help to build a better system for evaluating error

21 rates. In the current publishing culture in the field of cognitive neuroscience, as well as across

22 science generally, direct replications are discouraged. As a result, novel findings are seldom

23 replicated, which makes it hard to estimate the error rate. The open science movement has 
1 addressed this issue by emphasizing the value of direct replication (Zwaan et al., 2018). Thus, open science allows the development of a more cumulative science, and helps us evaluate the

3 error rate of a particular technique or method. In addition, open data itself provides greater

4 opportunity to evaluate methods and techniques. For example, the Consortium for Reliability and

5 Reproducibility (CoRR) has developed a range of useful resources to assess the reliability and

6 validity of many methods used in neuroimaging research (Poldrack et al., 2013; Zuo et al., 2014;

7 Zuo \& Xing, 2014).

8 Second, the open science movement may help to build a better peer review system, which

9 not only includes the usual pre-publication review but also a post-publication review (Earp \&

10 Wilkinson, 2017). Previously, the scientific community relied only on pre-publication peer-

11 review, which cannot fully evaluate the reliability and validity of a study since only the results

12 are visible to reviewers. The open science movement not only enhances the pre-publication

13 review, but also emphasize the value of post-publication review. The pre-publication review is

14 enhanced by pre-registration, open materials, scripts and data because in this case the evaluation

15 of a study is based on the entire research process. The post-publication review, which is

16 encouraged by the open science movement, also become valuable if the open scripts and open

17 data are made public along with the publication (e.g. http://www.russpoldrack.org/2013/04/how-

18 well-can-we-predict-future-criminal.html).

Third, the open science movement helps to build generally accepted standards which can

20 then lessen researcher flexibility (Gorgolewski et al., 2016; Pernet et al., 2018). Currently, nearly

21 every neuroimaging study uses its own workflow and its own standard for analyzing the

22 neuroimaging data (Carp, 2012; Yeung, 2018), and this granting of full flexibility to researchers

23 inflates the false positive rate. In the open science movement, a set of best practice is 
1 recommended (Nichols et al., 2017), and the whole field is beginning to establish a generally

2 accepted standard. These best practice standards may in time constitute a scientific routine that

3 helps experts to better evaluate the reliability of scientific evidence in the courtroom.

4 In sum, the open science movement increases the rigor and reproducibility of science,

5 and renders the whole research process more transparent to both researchers and the public. In

6 this way, open science may help scientists to become better gatekeepers for science, which in

$7 \quad$ turn will help judges to use the Daubert standard for better decision-making.

\section{Conclusion and Suggestions}

9 Based on the above analyses, we suggest that both cognitive neuroscientists and legal

10 practitioners would benefit from welcoming the open science movement. The open science

11 movement is not a simple standard, but "a set of beliefs, research practices, results, and policies

12 that are organized around the central roles of transparency and verifiability in scientific practice"

13 (Frank, 2018), involving a dynamic, upward spiral process. We suggest that both cognitive

14 neuroscientists and practitioners adjust their practices so that they are based on the best available 15 standards.

16 For cognitive neuroscientists, it is not easy to follow the newly established standards,

17 which require the preregistration of a study, complete reporting, and the opening of all data and

18 scripts. Fortunately, many practical guides have been published to help researchers navigate

19 through open science, e.g. (Hardwicke et al., 2018; Veldkamp et al., 2018). Professional

20 organizations have also begun to develop standards to enhance the rigor of science, e.g. the

21 Organization for Human Brain Mapping (OHBM) has created the Committee on Best Practices

22 in Data Analysis and Sharing (COBIDAS; http://www.humanbrainmapping.org/cobidas) to

23 guide analysis and sharing of MRI (Nichols et al., 2017) and M/EEG data (Pernet et al., 2018); 
1 while the American Psychological Association has also published standards for reporting both

2 quantitative (Appelbaum et al., 2018) and qualitative studies (Levitt et al., 2018). These practical

3 primers and standards provide researchers with a guide on how to do better as gatekeepers of

4 science.

5 For legal practitioners, it is crucial to be aware of the current irreproducibility problem

6 and its potential challenge to the credibility of scientific evidence. However, scientific journal

7 articles, including those on reproducibility and open science, are often too technical and cannot

8 be easily understood even by researchers, let alone legal practitioners (Tavris, 2014). Therefore,

9 to be better gatekeepers, it is important for legal practitioners to collaborate closely with

10 researchers. In neurolaw, there is a tradition of neuroscientists working closely with legal

11 scholars and legal practitioners. For example, in 2010, with the support of the MacArthur

12 Foundation, a group of neuroscientists edited a guidebook, A Judge's Guide to Neuroscience,

13 which explained the basics of neuroimaging studies in plain language (Bloom et al., 2010). It

14 would be helpful if there were similar guides for judges and other legal practitioners about

15 reproducibility and open science. If judges are made aware that published results can be false

16 positive and irreproducible, and that researchers may, intentionally or not, $p$-hack for publication,

17 they are likely to be more cautious when using scientific evidence from experts in their decision-

18 making. This awareness, further raising the bar set by the Daubert standard (Faigman \&

19 Monahan, 2009), may prevent additional unreliable scientific evidence from entering the

20 courtroom. 


\section{References}

Appelbaum, M., Cooper, H., Kline, R. B., Mayo-Wilson, E., Nezu, A. M., \& Rao, S. M. (2018). Journal article reporting standards for quantitative research in psychology: The APA Publications and Communications Board task force report. Am Psychol, 73(1), 3-25. doi:10.1037/amp0000191

Begley, C. G., \& Ellis, L. M. (2012). Drug development: Raise standards for preclinical cancer research. Nature, 483(7391), 531-533. doi:10.1038/483531a

Bloom, F. E., Fields, H. L., Gazzaniga, M. S., Grafton, S. T., Kiehl, K., Mayberg, H., . . . Wagner, A. (2010). A Judges Guide to Neuroscience. Retrieved from https://www.sagecenter.ucsb.edu/sites/staging.sagecenter.ucsb.edu/files/file-andmultimedia/A_Judges_Guide to_Neuroscience\%5Bsample\%5D.pdf

Boekel, W., Wagenmakers, E.-J., Belay, L., Verhagen, J., Brown, S., \& Forstmann, B. U. (2015). A purely confirmatory replication study of structural brain-behavior correlations. Cortex, 66, 115-133. doi:10.1016/j.cortex.2014.11.019

Buckholtz, J. W., \& Faigman, D. L. (2014). Promises, promises for neuroscience and law. Curr Biol, 24(18), R861-R867. doi:10.1016/j.cub.2014.07.057

Carp, J. (2012). On the plurality of (methodological) worlds: Estimating the analytic flexibility of fMRI experiments. Front Neurosci, 6. doi:10.3389/fnins.2012.00149

Chambers, C. D., Dienes, Z., McIntosh, R. D., Rotshtein, P., \& Willmes, K. (2015). Registered Reports: Realigning incentives in scientific publishing. Cortex, 66, A1-A2. doi:10.1016/j.cortex.2015.03.022 
1 Chen, X., Lu, B., \& Yan, C.-G. (2018). Reproducibility of R-fMRI metrics on the impact of different strategies for multiple comparison correction and sample sizes. Hum Brain Mapp, 39(1), 300-318. doi:10.1101/128645

Chin, J. M. (2014). Psychological science's replicability crisis and what it means for science in the courtroom. Psychology, Public Policy, and Law, 20(3), 225-238. doi:10.1037/law0000012

Christensen, A. M., Crowder, C. M., Ousley, S. D., \& Houck, M. M. (2014). Error and its Meaning in Forensic Science. J Forensic Sci, 59(1), 123-126. doi:10.1111/15564029.12275

Earp, B., \& Wilkinson, D. (2017). The publication symmetry test: a simple editorial heuristic to combat publication bias. Journal of Clinical and Translational Research, 3(S2), 5-7.

Faigman, D. L., \& Monahan, J. (2009). Standards of legal admissibility and their implications for psychological science. In J. L. Skeem, K. S. Douglas, \& S. O. Lilienfeld (Eds.), Psychological science in the courtroom: Consensus and controversy (pp. 3-25). New York: Guilford Press.

Farahany, N. A. (2016). Neuroscience and behavioral genetics in US criminal law: an empirical analysis. J Law Biosci, 2(3), 485-509. doi:10.1093/jlb/lsv059

Feresin, E. (2011). Italian court reduces murder sentence based on neuroimaging data. Retrieved from http://blogs.nature.com/news/2011/09/italian_court_reduces_murder_s.html

Frank, M. C. m. (2018, 24 Sep, 2018). What is "the open science movement"? It's a set of beliefs, research practices, results, and policies that are organized around the central roles of transparency and verifiability in scientific practice. Retrieved from https://twitter.com/mcxfrank/status/1044254887075147776 
1 Fusar-Poli, P., Radua, J., Frascarelli, M., Mechelli, A., Borgwardt, S., Di Fabio, F., . . David, S.

P. (2014). Evidence of reporting biases in voxel-based morphometry (VBM) studies of psychiatric and neurological disorders. Hum Brain Mapp, 35(7), 3052-3065. doi:10.1002/hbm.22384

Gazzaniga, M. S. (2008). The Law and Neuroscience. Neuron, 60(3), 412-415. doi:10.1016/j.neuron.2008.10.022

Gorgolewski, K. J., Auer, T., Calhoun, V. D., Craddock, R. C., Das, S., Duff, E. P., . . Poldrack, R. A. (2016). The brain imaging data structure, a format for organizing and describing outputs of neuroimaging experiments. Sci Data, 3, 160044. doi:10.1038/sdata.2016.44

Grove, W. M., Barden, R. C., Garb, H. N., \& Lilienfeld, S. O. (2002). Failure of RorschachComprehensive-System-based testimony to be admissible under the Daubert-JoinerKumho standard. Psychol Public Policy Law, 8(2), 216-234. doi:10.1037/10768971.8.2.216

Hardwicke, T. E., Mathur, M. B., MacDonald, K., Nilsonne, G., Banks, G. C., Kidwell, M. C., , . . Frank, M. C. (2018). Data availability, reusability, and analytic reproducibility: evaluating the impact of a mandatory open data policy at the journal Cognition. Royal Soc Open Sci, 5(8), 180448. doi:10.1098/rsos.180448

Hu, C.-P., Deng, X., Zhou, Z., \& Deng, X. (2011). Neurolaw: A marriage between young cognitive neuroscience and old law (in Chinese). Chin Sci Bull, 56(36), 3041-3053. doi:10.1360/972011-1911

Hu, C.-P., Kong, X.-Z., Wagenmakers, E.-J., Ly, A., \& Peng, K. (2018). Bayes Factor and Its Implementation in JASP: A Practical Primer (in Chinese). Adv Psychol Sci, 26(6), 951965. doi:10.3724/SP.J.1042.2018.00951 
1 Hu, C.-P., Wang, F., Guo, J., Song, M., Sui, J., \& Peng, K. (2016). The replication crisis in

psychological research (in Chinese). Adv Psychol Sci, 24(9), 1504-1518 doi:10.3724/SP.J.1042.2016.01504

Hughes, V. (2010). Science in court: Head case. Nature, 464, 340—342. doi:10.1038/464340a

Ioannidis, J. P. A., Munafò, M. R., Fusar-Poli, P., Nosek, B. A., \& David, S. P. (2014).

Publication and other reporting biases in cognitive sciences: detection, prevalence, and prevention. Trends Cogn Sci, 18(5), 235-241. doi:10.1016/j.tics.2014.02.010

Jones, O. D. (2004). Law, evolution and the brain: applications and open questions. Philos Trans Royal Soc B, 359(1451), 1697-1707. doi:10.1098/rstb.2004.1543

Levitt, H. M., Bamberg, M., Creswell, J. W., Frost, D. M., Josselson, R., \& Suárez-Orozco, C. (2018). Journal article reporting standards for qualitative primary, qualitative metaanalytic, and mixed methods research in psychology: The APA Publications and Communications Board task force report. Am Psychol, 73(1), 26-46. doi:10.1037/amp0000151

Liu, Y., Cheng, S., Fan, F., Di, X., Fan, H., Feng, C., . . Hu, C.-P. (2018). Reporting Standard for Meta-Analysis in Psychology: Status and Recommendations (in Chinese). ChinaXiv. Retrieved from http://www.chinaxiv.org/abs/201809.00177 doi:10.12074/201809.00177

Lyu, Z., Peng, K., \& Hu, C.-P. (2018). P-value, Confidence Intervals and Statistical Inference: A New Dataset of Misinterpretation. Front Psychol, 9, 868. doi:10.3389/fpsyg.2018.00868

Milham, M. P., Craddock, R. C., Son, J. J., Fleischmann, M., Clucas, J., Xu, H., . . Klein, A. (2018). Assessment of the impact of shared brain imaging data on the scientific literature. Nat Commun, 9(1), 2818. doi:10.1038/s41467-018-04976-1 
1 Munafò, M. R., Nosek, B. A., Bishop, D. V. M., Button, K. S., Chambers, C. D., Percie du Sert,

2 N., . . Ioannidis, J. P. A. (2017). A manifesto for reproducible science. Nat Hum Behav, l(1), 0021. doi:10.1038/s41562-016-0021

4 Nichols, T. E., Das, S., Eickhoff, S. B., Evans, A. C., Glatard, T., Hanke, M., .. Y Yeo, B. T. T. (2017). Best practices in data analysis and sharing in neuroimaging using MRI. Nat Neurosci, 20(3), 299-303. doi:10.1038/nn.4500

Nosek, B. A., Alter, G., Banks, G. C., Borsboom, D., Bowman, S. D., Breckler, S. J., . . . Yarkoni, T. (2015). Promoting an open research culture. Science, 348(6242), 1422-1425. doi:10.1126/science.aab2374

Nosek, B. A., Ebersole, C. R., DeHaven, A. C., \& Mellor, D. T. (2017). The Preregistration Revolution. Proc Natl Acad Sci USA.

12 Nosek, B. A., Ebersole, C. R., DeHaven, A. C., \& Mellor, D. T. (2018). The preregistration revolution. Proc Natl Acad Sci U.S.A., 115(11), 2600-2606. doi:10.1073/pnas. 1708274114

Open Science Collaboration. (2015). Estimating the reproducibility of psychological science.

17 Page, M., Taylor, J., \& Blenkin, M. (2011). Forensic Identification Science Evidence Since Daubert: Part I—A Quantitative Analysis of the Exclusion of Forensic Identification Science Evidence. J Forensic Sci, 56(5), 1180-1184. doi:10.1111/j.15564029.2011.01777.x

Pernet, C., Garrido, M., Gramfort, A., Maurits, N., Michel, C., Pang, E., . . Puce, A. (2018). Best Practices in Data Analysis and Sharing in Neuroimaging using MEEG. OSF Preprints. Retrieved from https://osf.io/a8dhx/ doi:10.31219/osf.io/a8dhx 
1 Poldrack, R. A., Baker, C. I., Durnez, J., Gorgolewski, K. J., Matthews, P. M., Munafo, M. R., . . . Yarkoni, T. (2017). Scanning the horizon: towards transparent and reproducible neuroimaging research. Nat Rev Neurosci, 18(2), 115-126. doi:10.1038/nrn.2016.167

4 Poldrack, R. A., Barch, D., Mitchell, J., Wager, T., Wagner, A., Devlin, J., . . Milham, M. (2013). Toward open sharing of task-based fMRI data: the OpenfMRI project. Front Neuroinform, 7(12). doi:10.3389/fninf.2013.00012

7 Saunders, B., Milyavskaya, M., Etz, A., Randles, D., \& Inzlicht, M. (2017). Reported self-

(1)
control is not meaningfully associated with inhibition-related executive function: A Bayesian analysis. PsyArXiv. Retrieved from https://psyarxiv.com/bxfsu/ doi:10.31234/osf.io/bxfsu

Shamseer, L., Moher, D., Clarke, M., Ghersi, D., Liberati, A., Petticrew, M., .. . Stewart, L. A. (2015). Preferred reporting items for systematic review and meta-analysis protocols (PRISMA-P) 2015: elaboration and explanation. BMJ, 349. doi:10.1136/bmj.g7647

Simmons, J. P., Nelson, L. D., \& Simonsohn, U. (2011). False-positive psychology: Undisclosed flexibility in data collection and analysis allows presenting anything as significant. Psychol Sci, 22(11), 1359-1366. doi:10.1177/0956797611417632

Szucs, D., \& Ioannidis, J. P. (2017). Empirical assessment of published effect sizes and power in the recent cognitive neuroscience and psychology literature. PLoS Biol, 15(3), e2000797. doi:10.1371/journal.pbio.2000797

Tavris, C. (2014). The Scientist-Practitioner gap: Revisiting “A View from the Bridge” a Decade Later. In S. O. Lilienfeld, S. J. Lynn, \& J. M. Lohr (Eds.), Science and PseudoScience in clinical Psychology (2 ed.). New York: The Guilford Press. 
1 Trafimow, D., \& Earp, B. D. (2017). Null hypothesis significance testing and Type I error: The domain problem. New Ideas in Psychology, 45, 19-27. doi:https://doi.org/10.1016/j.newideapsych.2017.01.002

Veldkamp, C., Bakker, M., Assen, M. v., Crompvoets, E., Ong, H., Nosek, B., . . Wicherts, J. (2018). Ensuring the quality and specificity of preregistrations. PsyArXiv.

Wagenmakers, E.-J., Wetzels, R., Borsboom, D., \& van der Maas, H. L. J. (2011). Why psychologists must change the way they analyze their data: the case of psi: comment on Bem (2011). J Pers Soc Psychol, 100(3), 426-432. doi:10.1037/a0022790

Westlye, L. T., Grydeland, H., Walhovd, K. B., \& Fjell, A. M. (2011). Associations between Regional Cortical Thickness and Attentional Networks as Measured by the Attention Network Test. Cereb Cortex, 21(2), 345-356. doi:10.1093/cercor/bhq101

Yeung, A. W. K. (2018). An Updated Survey on Statistical Thresholding and Sample Size of fMRI Studies. Front Hum Neurosci, 12(16). doi:10.3389/fnhum.2018.00016

Zuo, X.-N., Anderson, J. S., Bellec, P., Birn, R. M., Biswal, B. B., Blautzik, J., . . Milham, M. P. (2014). An open science resource for establishing reliability and reproducibility in functional connectomics. Sci Data, 1, 140049. doi:10.1038/sdata.2014.49

Zuo, X.-N., \& Xing, X.-X. (2014). Test-retest reliabilities of resting-state FMRI measurements in human brain functional connectomics: A systems neuroscience perspective. Neurosci Biobehav Rev, 45, 100-118. doi:10.1016/j.neubiorev.2014.05.009

Zwaan, R. A., Etz, A., Richard E., L., \& Donnellan, M. B. (2018). Making replication mainstream. Behav Brain Sci, 41, e120. doi:10.1017/S0140525X17001972 